\title{
Study of Eclipsing Binary and Multiple Systems in OB Associations IV: Cas OB6 Member DN Cas
}

\author{
V. Bakış ${ }^{1,3}$, H. Bakış ${ }^{1}$, S. Bilir ${ }^{2}$ and Z. Eker ${ }^{1}$ \\ ${ }^{1}$ Faculty of Science, Department of Space Sciences and Technologies, Akdeniz University, 07058, Antalya, Turkey \\ ${ }^{2}$ Faculty of Science, Department of Astronomy and Space Sciences, Istanbul University, 34119, Beyazit, Istanbul, Turkey \\ ${ }^{3}$ E-mail: volkanbakis@akdeniz.edu.tr
}

(ReCeived February 17, 2016; ACCEPTEd August 1, 2016)

\begin{abstract}
An early-type, massive, short-period $\left(P_{\text {orb }}=2^{d} .310951\right)$ eclipsing spectroscopic binary DN Cas has been re-visited with new spectral and photometric data. The masses and radii of the components have been obtained as $M_{1}=19.04 \pm$ $0.07 \mathrm{M}_{\odot}, M_{2}=13.73 \pm 0.05 \mathrm{M}_{\odot}$ and $R_{1}=7.22 \pm 0.06 \mathrm{R}_{\odot}, R_{2}=5.79 \pm 0.06 \mathrm{R}_{\odot}$, respectively. Both components present synchronous rotation $\left(V_{\text {rot1 }}=160 \mathrm{~km} \mathrm{~s}^{-1}, V_{\text {rot2 }}=130 \mathrm{~km} \mathrm{~s}^{-1}\right)$ with their orbit. Orbital period analysis yielded a physically bound additional component in the system with a minimum mass of $M_{3}=0.88 \mathrm{M}_{\odot}$ orbiting in an eccentric orbit $(e=0.37 \pm 0.2)$ with an orbital period of $P_{12}=42 \pm 9 \mathrm{yr}$. High precision absolute parameters of the system allowed us to derive a distance to DN Cas as $1.7 \pm 0.2 \mathrm{kpc}$ which locates the system within the borders of the Cas OB6 association $(d=1.8 \mathrm{kpc})$. The space velocities and the age of DN Cas are in agreement with those of Cas OB6. The age of DN Cas ( $\tau=3-5 \mathrm{Myr}$ ) is found to be 1-2 Myr older than the embedded clusters (IC 1795, IC 1805, and IC 1848) in the Cas OB6 association, which implies a sequential star formation in the association.
\end{abstract}

Keywords: galaxy: open clusters and associations: individual: Cas OB6 - stars: early-type - stars: individual: DN Cas

\section{INTRODUCTION}

OB associations host the gravitationally unbound youngest and massive stars in the galaxy; therefore, stellar parameters of the members of OB associations help understanding their structure and evolution. The most powerful technique to obtain age, chemical properties, and a reliable distance is doubtlessly the use of eclipsing binaries, which are the members of the association. Luckily, the relative number of eclipsing binary and multiple (EBM) systems in OB associations is high (e.g. Mason et al. 1998). Within the scope of investigating EBM systems in OB associations with modern analysis techniques (i.e. Bakış et al. 2015), an early-type massive binary DN Cas in the direction of Cas OB6 complex is selected for a more detailed study than available in the literature today.

Located in the front edge of the Perseus spiral arm, the Cas OB6 association is one of the most outstanding associations in the Cas OB complex (see Figure 1). Its distance and mean radial velocity were determined by Mel'nik \& Dambis (2009) as $1.8\left(\mathrm{kpc}\right.$ ) and $-42.7 \pm 8.2 \mathrm{~km} \mathrm{~s}^{-1}$, respectively. The association hosts a number of $\mathrm{H}$ II regions (IC 1805, IC 1848, S196, and S201) and several radio sources W5 (IC 1848), W4 (surrounding IC 1805), and the W3 region (IC 1795). These regions are known with massive stellar content and active stellar formation which goes back in time to 3-5 Myr for IC 1795 (Bik et al. 2012), 1-3 Myr for IC 1805 (Straižys et al. 2013), and 3-5 Myr for IC 1848 (Lim et al. 2014).

DN Cas is located within the borders of Cas OB6. Its photometric variation was discovered by Hoffmeister (1947) and has been observed photometrically by Frazier \& Hall (1974) for the first time. Due to variability of the selected comparison star, Frazier \& Hall (1974) was not able to solve the multi-band light curves. Davidge (1980) performed $U B V$ photometry of the system without the light curve solution. Zakirov (2001) who investigated the relationship of DN Cas with Cas OB6 made estimations on the absolute parameters of the companions using his own photometric data in $U B V R I$ bands . However, due to absence of the spectral information, the estimated parameters were not consistent with the spectral types of the components.

The most recent study on DN Cas is the radial velocity survey of the 14 association members of Cas OB6 (Hillwig et al. 2006). They reported a binarity of $50 \%$ in their sample and obtained the spectroscopic orbit of DN Cas for the first time. Using the orbital parameters from the analysis of incomplete monochromatic light curve of DN Cas, stellar parameters of the components of DN Cas were estimated. 


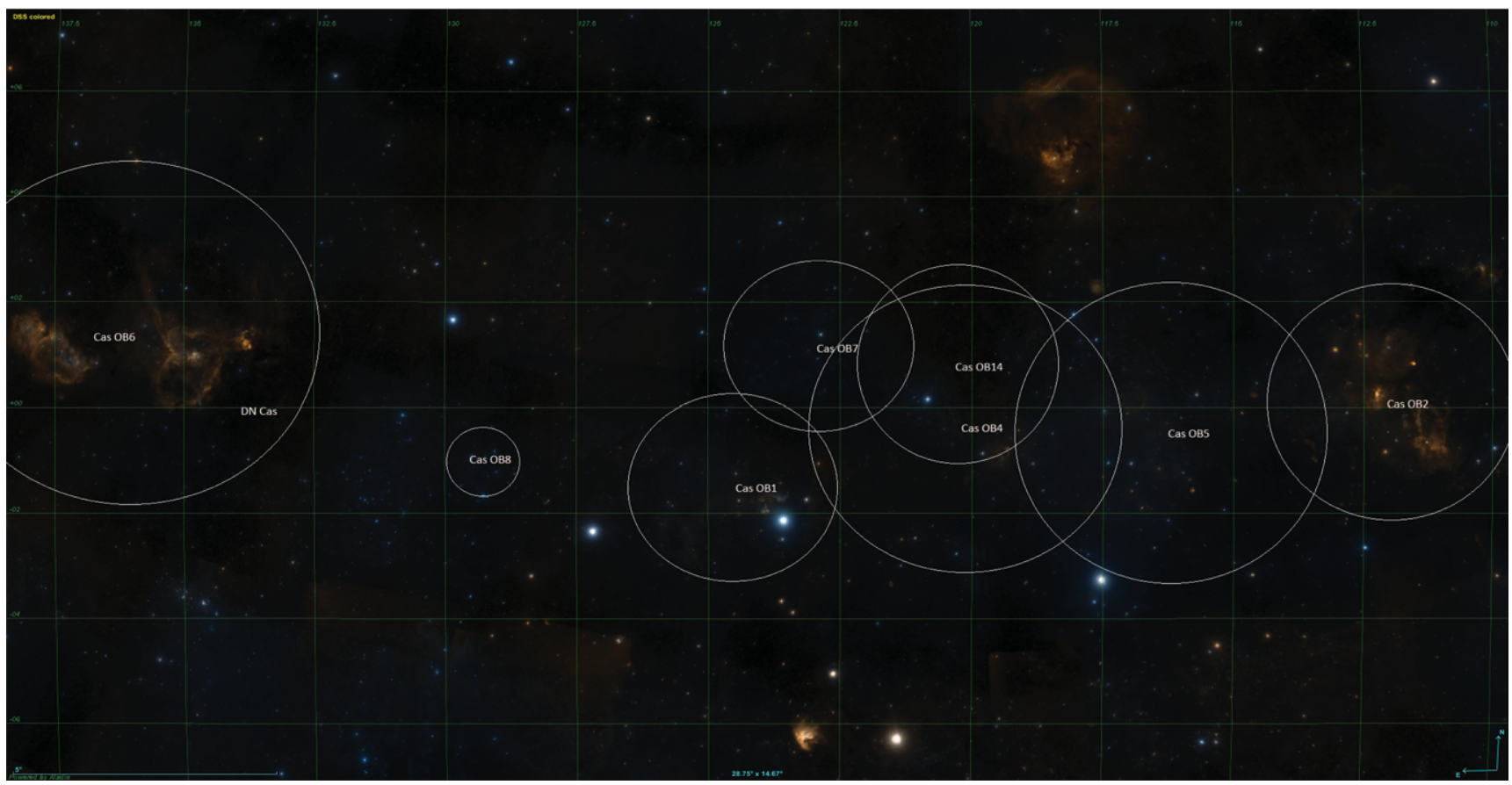

Figure 1. The distribution of the Cas OB associations towards galactic coordinates $l=120.3^{\circ}$ and $b=-0.9^{\circ}$, created with the Aladin Sky Atlas (Bonnarel et al. 2000). The borders of individual associations were determined using the catalogue data of Meln'ik and Dambis (2009) and Tetzlaff et al. (2010).

However, necessity of multi-band photometric observations of the system was announced probably due to the need to clarify the inconsistency of the mass and spectral type of the components they derived.

\section{OBSERVATIONS}

\subsection{Photometry}

There are three sources of photometric data of DN Cas used in this research: The first set of photometric data comes from the observations of Zakirov (2001) which is not available in the literature. The observations of Zakirov (2001) were used in this paper for only orbital period study (Section 3). The second set of photometric data was collected from the observations which were performed in Çanakkale Onsekiz Mart University Ulupinar Observatory in 2009 with 30 - and $40-\mathrm{cm}$ Cassegrain-Schmidt type telescopes equipped with $1 \mathrm{k} \times 1 \mathrm{k}$ CCDs and Bessell $U B V R_{c} I_{c}$ filters. A total of 4956 images have been collected in seven nights. The data obtained in this campaign was not evenly distributed in phase; therefore, they were used for only orbital period analysis with a new time of minimum (HJD 2455128.3279 \pm 0.0005 ) (see Section 3).

The third set of photometric data was collected during the observing campaign in Akdeniz University Campus Telescope (AUCT) in 2012 using a 25-cm Ritchey-Chretien type telescope equipped with a back-illuminated $1 \mathrm{k} \times 1 \mathrm{k} \mathrm{CCD}$ camera and Bessell $U B V R_{c} I_{c}$ filters. A total of $3840 \mathrm{im}-$ ages has been collected in nine nights. The number of data and its distribution are enough to analyse the multi-band light curves of DN Cas. The mean standard deviation of the observations are $0.033,0.011,0.009,0.007$, and 0.010 in $U B V R_{c}$ and $I_{c}$ filters, respectively. The comparison star HD 14558 was selected for differential photometry and the check star was SAO 12245. The reduction of the photometric data is standard for CCD photometry; bias and dark subtraction and flat field correction. The photometry of the reduced data were performed by means of aperture photometry where the star aperture was selected to be three times the FWHM of the stellar profile. A new time of primary minimum has been extracted from the AUCT observations (HJD 2456287.2716 \pm 0.0003 ).

\subsection{Spectroscopy}

The spectroscopic observations has been obtained in 2013 with the intermediate resolution ( $\mathrm{R} \sim 5$ 100) TUG Faint Object Spectrograph and Camera (TFOSC) attached to 1.5m RTT150 telescope of TÜBITAK National Observatory ${ }^{1}$, Turkey. TFOSC provides a wavelength coverage from $\lambda=$ 330 to $1200 \mathrm{~nm}$ in 11 spectral orders. A total of 20 spectra have been obtained with TFOSC. The reduction of TFOSC spectra has been made in Image Reduction and Analysis Facility $\left(\mathrm{IRAF}^{2}\right)$ software . The journal of spectroscopic observations is given in Table 1 and two spectral orders obtained at three different epochs are shown in Figure 2. The spectral data obtained in this study were used as complementary to the existing radial velocity data of Hillwig et al. (2006).

\footnotetext{
${ }^{1}$ http://www.tug.tubitak.gov.tr/

${ }^{2}$ IRAF is distributed by the National Optical Astronomy Observatories.
} 
Table 1. Journal of spectroscopic observations and radial velocities (RV) of the components.

\begin{tabular}{rcccccc}
\hline \hline Number & $\begin{array}{c}\text { Date } \\
\text { HJD-2400000 }\end{array}$ & Orbital phase & $\begin{array}{c}\text { Exposure time } \\
(\mathrm{s})\end{array}$ & S/N & $\begin{array}{c}\text { Pri RV } \\
\left(\mathrm{km} \mathrm{s}^{-1}\right)\end{array}$ & $\begin{array}{c}\text { Sec RV } \\
\left(\mathrm{km} \mathrm{s}^{-1}\right)\end{array}$ \\
\hline 1 & 56303.4372 & 0.997 & 1200 & 110 & -66 & - \\
2 & 56306.2212 & 0.202 & 1200 & 200 & -289 & 228 \\
3 & 56306.2386 & 0.209 & 1200 & 160 & -302 & 182 \\
4 & 56648.2582 & 0.209 & 300 & 110 & -277 & 196 \\
5 & 56648.2642 & 0.211 & 300 & 110 & -276 & 193 \\
6 & 56648.2717 & 0.214 & 300 & 110 & -278 & 185 \\
7 & 56649.2205 & 0.625 & 300 & 160 & 70 & -267 \\
8 & 56649.2307 & 0.629 & 300 & 140 & 51 & -306 \\
9 & 56649.2403 & 0.633 & 300 & 140 & 61 & -297 \\
10 & 56649.2500 & 0.637 & 300 & 130 & 61 & -311 \\
11 & 56649.2588 & 0.641 & 300 & 130 & 68 & -343 \\
12 & 56649.2681 & 0.645 & 300 & 150 & 60 & -310 \\
13 & 56649.2762 & 0.649 & 300 & 130 & 35 & -349 \\
14 & 56649.2856 & 0.653 & 300 & 100 & 75 & -327 \\
15 & 56649.2946 & 0.657 & 300 & 90 & 104 & -318 \\
16 & 56649.3027 & 0.660 & 300 & 50 & 116 & -273 \\
17 & 56649.3223 & 0.669 & 300 & 70 & 159 & -253 \\
18 & 56649.3303 & 0.672 & 300 & 70 & 108 & -371 \\
19 & 56649.3522 & 0.682 & 300 & 90 & 128 & -304 \\
20 & 56649.3604 & 0.685 & 300 & 100 & 130 & -353 \\
\hline \hline
\end{tabular}
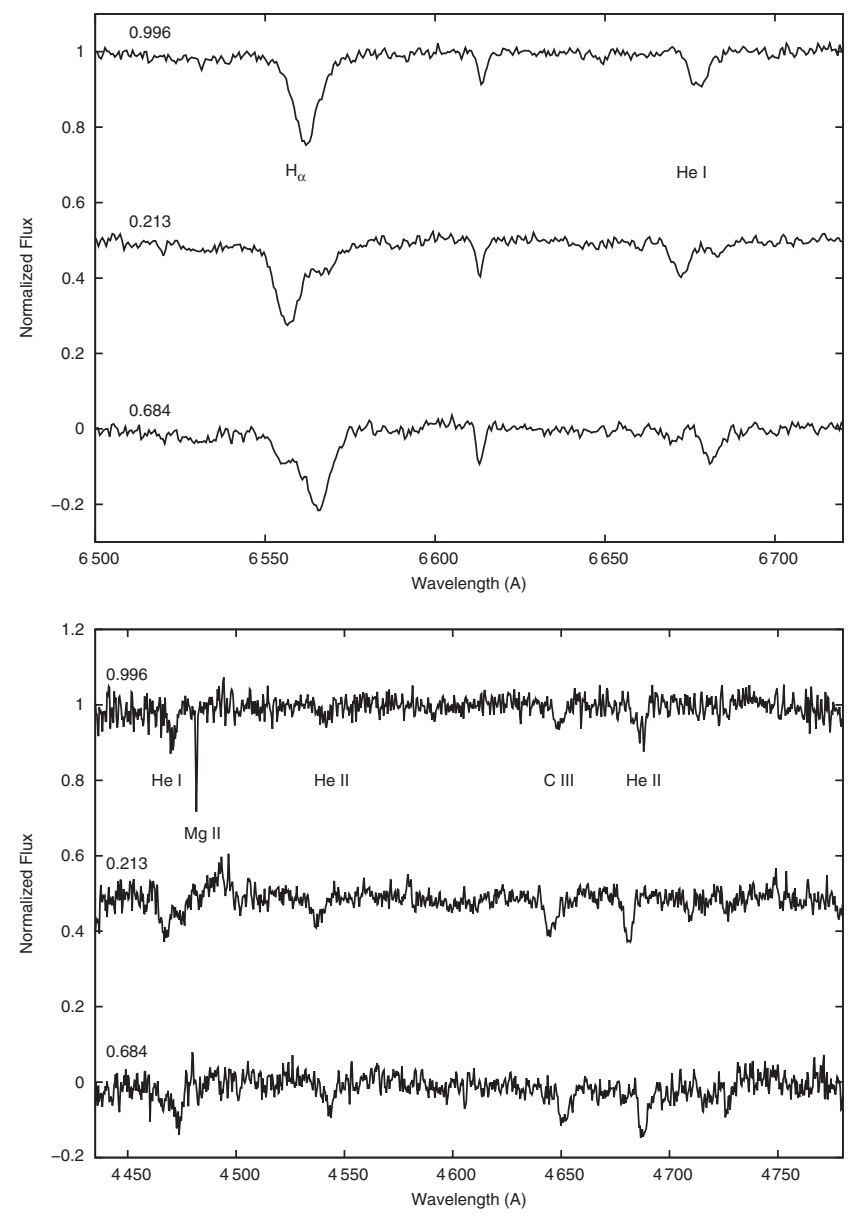

Figure 2. Two spectral orders obtained at three different orbital phases.
The spectral lines of the components of DN Cas are well separated (see Figure 2) which allowed us to measure the spectral lines of the individual components reliably. The radial velocities of DN Cas were measured by means of fitting Gaussian to the line centres using the SPLOT task in IRAF. The uncertainties of the radial velocity measurements have been determined as the standard deviation of three consecutive measurements which is on the order of $2-3 \mathrm{~km} \mathrm{~s}^{-1}$. However, we estimate overall radial velocity uncertainty in the range $5-9 \mathrm{~km} \mathrm{~s}^{-1}$ due to the low resolution of the spectrograph. The measured radial velocities are given in Table 1 .

\section{ORBITAL PERIOD VARIATION}

The best tool to analyse the orbital period variation is the $\mathrm{O}-\mathrm{C}$ analysis. The available times of minima data of DN Cas were collected from the literature and listed in Table 2 together with those determined in this study. The $\mathrm{O}-\mathrm{C}$ residuals in Table 2 were calculated with the following light elements (Kreiner, Kim, \& Nha 2001):

$$
\mathrm{HJD}_{\min }=2441388.5662+2^{d} .31095111 \times E,
$$

where $\mathrm{HJD}_{\min }$ and $E$ are the heliocentric time of conjunction for the primary minimum and the epoch number, respectively.

As listed in Table 2, the complete data set consists of 1 visual (vis), 21 photographic ( $p g), 7$ photoelectric (pe), and $16 c c d$ times of minima. The visual and photographic observations are relatively less accurate; therefore, the weighting scheme was chosen as follows: vis and $p g=1$, pe and $c c d=10$.

The $\mathrm{O}-\mathrm{C}$ residuals are plotted in Figure 3. The relative accuracy of the pe and $c c d$ data except in a few case is 
Table 2. Times of minima of DN Cas.

\begin{tabular}{|c|c|c|c|c|c|c|c|}
\hline $\begin{array}{l}\text { Times of minima } \\
\text { HJD - } 2400000\end{array}$ & Method & Type & $\mathrm{O}-\mathrm{C}$ & $\begin{array}{c}\text { Times of minima } \\
\text { HJD - } 2400000\end{array}$ & Method & Type & $\mathrm{O}-\mathrm{C}$ \\
\hline 28180.3100 & $\mathrm{pg}$ & $\mathrm{sec}$ & -0.0151 & 48172.3636 & pe & $\mathrm{sec}$ & 0.0004 \\
\hline 28427.6000 & pg & $\mathrm{sec}$ & 0.0031 & 48619.5316 & pe & pri & -0.0006 \\
\hline 28835.5100 & pg & pri & 0.0302 & 49615.5498 & pe & pri & -0.0024 \\
\hline 28865.4200 & pg & pri & -0.1021 & 50842.6600 & ccd & pri & -0.0072 \\
\hline 29103.4800 & $\mathrm{pg}$ & pri & -0.0701 & 51107.2662 & pe & $\mathrm{sec}$ & -0.0049 \\
\hline 29163.5900 & pg & pri & -0.0448 & 51422.7160 & ccd & pri & 0.0001 \\
\hline 29646.6500 & pg & pri & 0.0264 & 51466.6180 & ced & pri & -0.0060 \\
\hline 29697.4800 & pg & pri & 0.0155 & 52587.4405 & ced & pri & 0.0052 \\
\hline 29957.4700 & pg & $\mathrm{sec}$ & 0.0235 & 53314.2254 & ced & $\mathrm{sec}$ & -0.0040 \\
\hline 30023.4000 & pg & pri & 0.0914 & 53657.4062 & ccd & pri & 0.0006 \\
\hline 30321.3800 & pg & pri & -0.0413 & 53980.9378 & ccd & pri & -0.0010 \\
\hline 30372.2600 & pg & pri & -0.0023 & 54050.2669 & ced & pri & -0.0004 \\
\hline 30582.5300 & pg & pri & -0.0288 & 54066.4437 & $\mathrm{ccd}$ & pri & -0.0003 \\
\hline 30590.5500 & pg & $\mathrm{sec}$ & -0.0971 & 54838.3062 & $\mathrm{ccd}$ & pri & 0.0045 \\
\hline 39024.4840 & pg & pri & 0.0208 & 55087.8900 & ccd & pri & 0.0056 \\
\hline 39053.3940 & pg & $\mathrm{sec}$ & 0.0439 & 55872.4607 & ccd & $\mathrm{sec}$ & 0.0084 \\
\hline 39061.4030 & pg & pri & -0.0354 & 55879.3873 & pe & $\mathrm{sec}$ & 0.0022 \\
\hline 41388.5710 & pg & pri & 0.0048 & 55880.5433 & pe & pri & 0.0027 \\
\hline 41602.3650 & pg & sec & 0.0358 & 55903.6532 & ced & pri & 0.0031 \\
\hline 44191.7650 & pg & pri & 0.0151 & 55953.3388 & ced & $\mathrm{sec}$ & 0.0032 \\
\hline 45044.4772 & pg & pri & -0.0137 & 55128.3279 & ccd & $\mathrm{sec}$ & 0.0019 \\
\hline 45653.4610 & vis & sec & 0.0345 & 56287.2716 & ccd & pri & 0.0036 \\
\hline 47928.5576 & pe & pri & -0.0002 & & & & \\
\hline
\end{tabular}

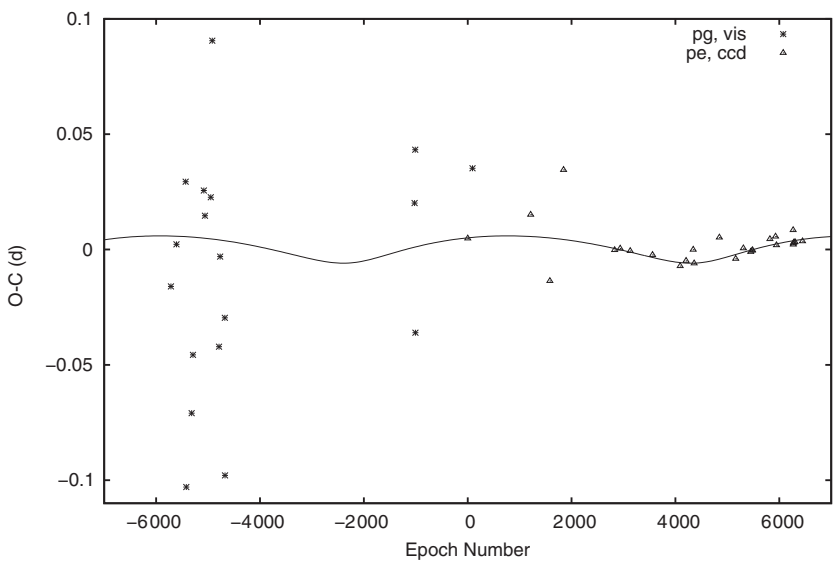

Figure 3. $\mathrm{O}-\mathrm{C}$ residuals and the best fitting LTE orbit.

noteworthy. The residuals after the epoch number 2000 clearly shows a cyclic change which can be modelled by the light time effect (LTE) caused by gravitational disturbance of a distant companion to DN Cas. Irwin (1959) derived the following equation [Equation (2] to represent the $\mathrm{O}-\mathrm{C}$ variations in the case of an existence of a distant companion:

$$
\Delta t=\frac{a_{12}^{\prime} \sin i^{\prime}}{c}\left\{\frac{1-e^{\prime 2}}{1+e^{\prime} \cos v^{\prime}} \sin \left(v^{\prime}+\omega^{\prime}\right)+e^{\prime} \cos \omega^{\prime}\right\},
$$

where $a_{12}^{\prime}, i^{\prime}, e^{\prime}$, and $\omega^{\prime}$ are the semi-major axis, orbital inclination, eccentricity, and the argument of periastron of the long orbit (AB), respectively. Analysing the times of minima data with Equation (2) yielded the orbital period of the wide
Table 3. Parameters of the wide orbit from the $\mathrm{O}-\mathrm{C}$ analysis.

\begin{tabular}{lc}
\hline \hline Parameter & Value \\
\hline$T^{\prime}(\mathrm{HJD})$ & $2451700(280)$ \\
$P_{12}(\mathrm{yr})$ & $42(9)$ \\
$a_{12}^{\prime} \sin i^{\prime}(\mathrm{AU})$ & $1.03(0.13)$ \\
$e^{\prime}$ & $0.37(0.2)$ \\
$\omega^{\prime}(\mathrm{rad})$ & $5.0(0.5)$ \\
$f\left(M_{3}\right)\left(\mathrm{M}_{\odot}\right)$ & $0.0006(0.0002)$ \\
\hline \hline
\end{tabular}

orbit as $P_{12}=42(9) \mathrm{yr}$ with an eccentricity of $0.37(0.2)$. Other orbital parameters of the distant companion are listed in Table 3. The best fitting LTE model is shown in Figure 3.

\section{ANALYSIS OF THE PHOTOMETRIC AND RADIAL VELOCITY DATA}

The photometric data in the $U B V R_{c}$ and $I_{c}$ bands have been analysed together with the radial velocity data of DN Cas using the 2007 version of the Wilson-Devinney (WD) code (Wilson \& Devinney 1971). The initial values of the input parameters such as the orbital inclination, mass-ratio, and relative radii of the components were adopted from the work of Hillwig et al. (2006). The orbital period and the conjunction time were adopted from the orbital period analysis (Section 3) and fixed during the solution. The albedo and 
Table 4. Results from the simultaneous solution of light curves of DN Cas. Adjusted and fixed parameters are presented in the separate panels of the table. Uncertainties of the adjusted parameters, as suggested by WD code, are given in brackets.

\begin{tabular}{|c|c|c|}
\hline Parameter & Symbol & Value \\
\hline Heliocentric primary conjunction (HJD-240000) & $T_{0}$ & $56287.2699(0.0003)$ \\
\hline Effective temperature (pri)(K) & $T_{\text {eff1 }}$ & 32100 \\
\hline Effective temperature $(\mathrm{sec})(\mathrm{K})$ & $T_{\text {eff2 }}$ & $28020(100)$ \\
\hline Light contribution (pri) in $U$-band & $L_{1} / L_{1+2}(U)$ & $0.666(0.006)$ \\
\hline Light contribution (pri) in $B$-band & $L_{1} / L_{1+2}(B)$ & $0.658(0.006)$ \\
\hline Light contribution (pri) in $V$-band & $L_{1} / L_{1+2}(V)$ & $0.657(0.006)$ \\
\hline Light contribution (pri) in $R_{c}$-band & $L_{1} / L_{1+2}\left(R_{c}\right)$ & $0.662(0.006)$ \\
\hline Light contribution (pri) in $I_{c}$-band & $L_{1} / L_{1+2}\left(I_{c}\right)$ & $0.658(0.006)$ \\
\hline Orbital inclination $\left(^{\circ}\right)$ & $i$ & $77.2(0.2)$ \\
\hline Mass ratio $\left(M_{2} / M_{1}\right)$ & $q$ & $0.722(0.002)$ \\
\hline Systemic velocity $\left(\mathrm{km} \mathrm{s}^{-1}\right)$ & $V_{\gamma}$ & $-45.5(0.3)$ \\
\hline Surface potential (pri) & $\Omega_{1}$ & $4.03(0.01)$ \\
\hline Surface potential (sec) & $\Omega_{2}$ & $4.11(0.03)$ \\
\hline First critical Roche potential & $\Omega_{\mathrm{cr}}$ & 3.28 \\
\hline Relative radii (pri) & $r_{1}$ & $0.307(0.002)$ \\
\hline Relative radii ( $\mathrm{sec}$ ) & $r_{2}$ & $0.246(0.002)$ \\
\hline \multicolumn{3}{|l|}{ Fixed parameters: } \\
\hline Orbital period (d) & $P(\mathrm{~d})$ & 2.310951 \\
\hline Orbital eccentricity & $e$ & 0.0 \\
\hline Rotation rate & $F$ & 1.0 \\
\hline
\end{tabular}

gravity darkening coefficients were set to 1.0 as suggested for early type stars. An infrared light source near DN Cas with an angular distance of 5.3 arcsec has been noted by Hillwig et al. (2006), which is in the area covered by our photometric aperture. Hence, the third light source has been considered and the third light contribution $\left(l_{3}\right)$ has been converged during the solution. The orbital eccentricity was also taken into account and it was set as a free parameter during the first iterations.

The WD code converged and found the best fitting model parameters after a few iterations. Initial system parameters were altered and the data were re-analysed to check if the solution is stable and hence, final parameter set is reliable. Only a small value found for the eccentricity ( $e=0.001$ ) was doubtful. However, its relatively big uncertainty $\Delta e=0.003$ decreased its reliability and allowed us to adopt a circular orbit although the system DN Cas did not still complete its circularisation time scale yet (see Section 5.3). Nevertheless, it should be noted that the effect of such a small eccentricity on the determination of the physical parameters of the components is negligible.

In order to check the synchronicity of the components with the orbit, we modelled the spectral lines of the components and it is found that they rotate synchronously with the orbit (see Table 5). In the WD code, this synchronous rotation is stated by fixing the $F$ parameter to 1.0 for each parameter to express the rotation rate of the component star.

The shapes of the components of close binary systems departs from sphere due to tidal effects causing a shift of the light centres from the mass centre of the components. Due to the difference between the light centres and the mass cen- tres of the component stars, the spectroscopic orbital model does not represent the true orbit (see the study by Özkardeş, Erdem, \& Bakış (2009) on the highly distorted star CN Hyi). In the case of DN Cas, both stars are well inside their roche lobes (see Table 4) and therefore nearly spherical. The final set of light curve and spectroscopic orbital parameters are listed in Table 4 and the model light curves together with the spectroscopic orbit are shown in Figure 4. The system is detached and both components were found to be well inside their roche lobes.

The third light contribution was found to be negligible in all bands. As calculated by Hillwig et al. (2006), the light contribution of the third star is $14 \%$ at $H \& K$ infrared bands. This means that the light contribution of the third light source is not detectable at the $I$-band. The nature of the third light source is discussed in Section 5.2.

\section{DISCUSSION}

\subsection{Astrophysical parameters and the distance of the system}

The simultaneous solution of the light curve and the radial velocity data allowed us to obtain the physical parameters of the components of DN Cas, which are presented in Table 5. The masses and radii of the components are derived with an uncertainty of $\sim 0.5 \%$. The masses of the components derived in this study is $\sim 1 \%$ smaller than the masses derived by Hillwig et al. (2006), which is in the uncertainty box given for their mass determinations. Following the tables of stellar parameters given by Straižys \& Kurilene (1981), the physical 

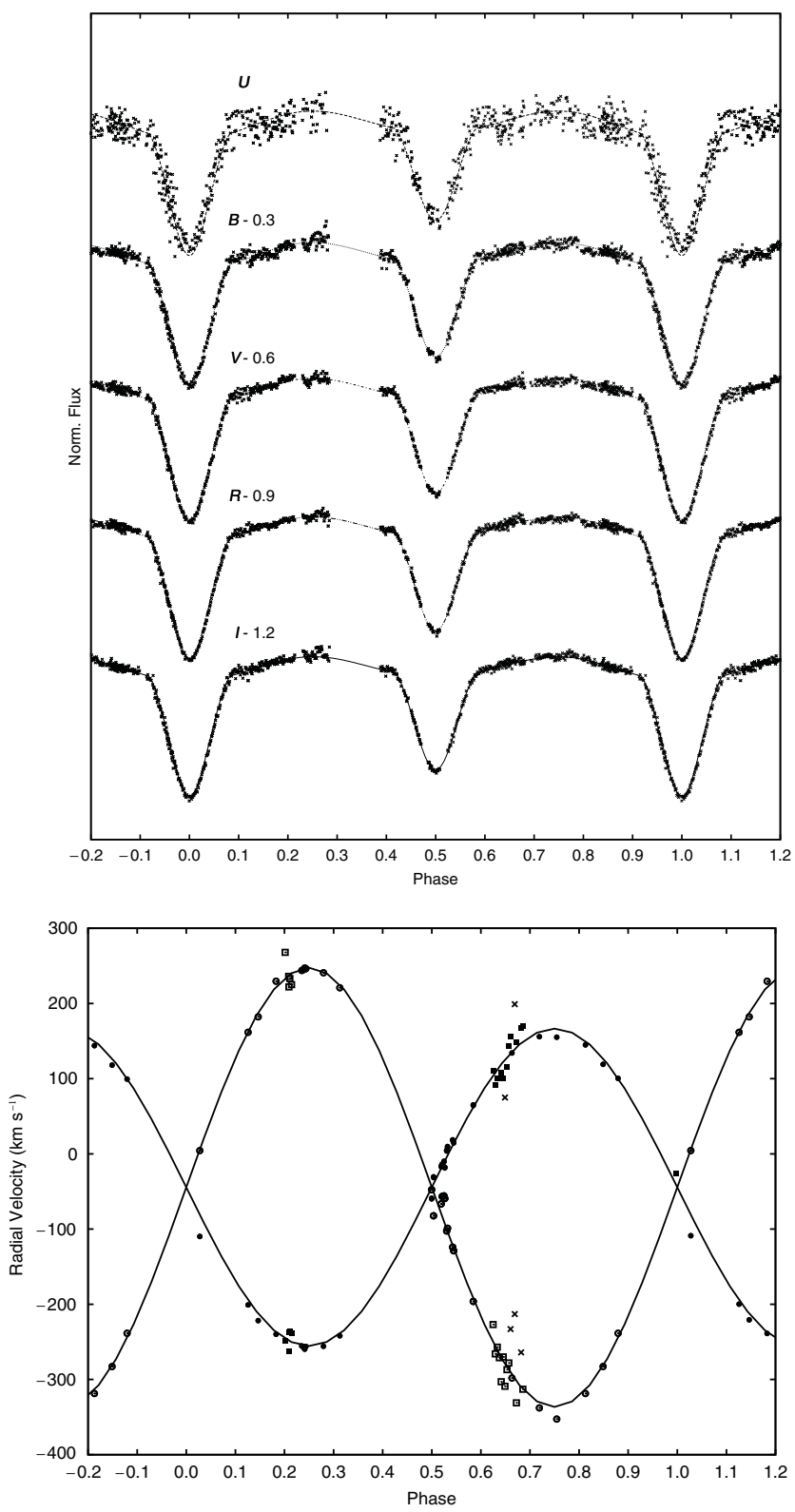

Figure 4. Light curve (top) and spectroscopic orbit (bottom) models. The radial velocities shown by filled and empty circles are from Hillwig et al. (2006) and the filled and empty squares are obtained in this study. Radial velocities shown with crosses were not included in the analysis.

parameters of the components imply that the spectral types of the primary and secondary components are B0V and B1V.

The distance of the system is calculated by deriving the unreddened colour indices of the components with the $Q$ method of Johnson \& Morgan (1953). The unreddened colour indices allowed us to derive the interstellar extinction of $A_{v}=3^{m} .1(0.03)$. The absolute visual magnitude of the components has been derived by using the bolometric magnitude and the bolometric correction. Visual magnitude of the system, interstellar extinction, and the absolute visual magnitude of the components has been used in the distance modulus to derive the distance of $1740 \pm 220 \mathrm{pc}$ for the system.
The rotational velocities of the components were also measured by means of fitting synthetic spectra to the spectral lines of the components. The model atmosphere parameters were adopted from Table 5 and the suitable model atmospheres were selected from the Kurucz/Grids of model atmospheres $^{3}$. Modelling the spectral lines showed that both components rotate $\left(V_{\text {rot1 }}=160 \mathrm{kms}^{-1}, V_{\text {rot2 }}=130 \mathrm{kms}^{-1}\right)$ in accordance with their synchronisation rotational velocities given in Table 5 .

\subsection{The unseen third companion}

The orbital period analysis of the system (Section 3) yielded a cyclic period change, which was modelled with LTE orbit of $P_{12}=42(9)$ yr. The mass function for this triple system is $f\left(m_{3}\right)=a_{12}^{\prime} \sin i^{\prime} / P_{12}=0.0006 \pm 0.0002 \mathrm{M}_{\odot}$. Assuming the inclination of the third component's orbit is $90^{\circ}$, the mass of the third star is found to be $0.88 \mathrm{M}_{\odot}$. In case, the orbit of the third star is co-planar $\left(i=i^{\prime}=77.2^{\circ}\right)$ with the close binary system, the mass of the third star is $0.90 \mathrm{M}_{\odot}$. If we assume a main-sequence star for the third companion, it should be a K0 type star and its light ratio in a system with B0 and B1 stars is much less than $1 \%$. Low light contribution of the third star can explain its invisibility in the visible region of the light curve and spectra.

\subsection{Evolutionary status of DN Cas}

The evolutionary status of the components of DN Cas have been investigated by locating the components in the HRdiagram together with the evolutionary tracks and isochrones of Bertelli et al. (2009) (see Figure 5). The chemical composition of DN Cas is not known, therefore, the isochrones in Figure 5 have been calculated for two metallicity values ( $Z=0.02$ and 0.04 ) in order to set the lower and upper limits of the age. Moreover, we have plotted the members of IC 1805 and IC 1848 open clusters in the same plane in Figure 5 to see if there is an age difference. The best fitting isochrones yielded an age of $\tau=3 \mathrm{Myr}$ for $Z=0.04$ and $\tau=5 \mathrm{Myr}$ for $Z=0.019$ with and uncertainty of $\pm 1 \mathrm{Myr}$. Isochrones in Figure 5 imply that the members of the two open clusters are 1-2 Myr younger than DN Cas.

According to Zahn (1977), close binary systems tend to circularise their orbit due to tidal effects. Following his mathematical formulation, the circularisation time scale for DN Cas is $\sim 22 \pm 0.2 \mathrm{Myr}$. This circularisation time scale is apparently bigger than the age of the system $(\tau=3-5$ Myr) but the difference is small compared to stellar evolution. This may imply a possible eccentricity for the orbit. However, the light curve and spectroscopic orbit analysis yielded a very small eccentricity $(e=0.001)$ with a relatively big uncertainty box $(\Delta e=0.003)$. Moreover, we do not know the effect of the third star on the orbit circularisation. Therefore, higher quality observational data

\footnotetext{
${ }^{3}$ http://kurucz.harvard.edu/grids.html
} 
Table 5. Close binary stellar parameters of DN Cas. Errors of parameters are given in brackets.

\begin{tabular}{|c|c|c|c|}
\hline Parameter & Symbol & Primary & Secondary \\
\hline Spectral type & $\mathrm{Sp}$ & $\mathrm{B} 0 \mathrm{~V}$ & $\mathrm{~B} 1 \mathrm{~V}$ \\
\hline $\operatorname{Mass}\left(\mathrm{M}_{\odot}\right)$ & $M$ & $19.04(0.07)$ & $13.73(0.05)$ \\
\hline Radius $\left(\mathrm{R}_{\odot}\right)$ & $R$ & $7.22(0.06)$ & $5.79(0.06)$ \\
\hline Separation $\left(\mathrm{R}_{\odot}\right)$ & $a$ & \multicolumn{2}{|c|}{$23.53(0.05)$} \\
\hline Orbital period (d) & $P$ & \multicolumn{2}{|c|}{$2.310951(0.000001)$} \\
\hline Orbital inclination $\left(^{\circ}\right)$ & $i$ & \multicolumn{2}{|c|}{$77.2(0.2)$} \\
\hline Mass ratio $\left(M_{2} / M_{1}\right)$ & $q$ & \multicolumn{2}{|c|}{$0.722(0.002)$} \\
\hline Eccentricity & $e$ & \multicolumn{2}{|c|}{0.0} \\
\hline Surface gravity (cgs) & $\log g$ & $4.000(0.009)$ & $4.270(0.010)$ \\
\hline Colour index (mag) & $B-V$ & \multicolumn{2}{|c|}{$0.70(0.01)$} \\
\hline Colour excess (mag) & $E(B-V)$ & \multicolumn{2}{|c|}{$1.00(0.01)$} \\
\hline Intrinsic colour index (mag) & $(B-V)_{0}$ & \multicolumn{2}{|c|}{$-0.30(0.01)$} \\
\hline Visual absorption (mag) & $A_{\mathrm{v}}$ & \multicolumn{2}{|c|}{$3.10(0.03)$} \\
\hline Component visual magnitude (mag) & $V_{1,2}$ & $7.29(0.03)$ & $7.99(0.04)$ \\
\hline Temperature (K) & $T_{\text {eff }}^{1,2}$ & $32100(1000)$ & $28020(1100)$ \\
\hline Luminosity $\left(\mathrm{L}_{\odot}\right)$ & $\log L$ & $4.70(0.06)$ & $4.27(0.08)$ \\
\hline Bolometric magnitude (mag) & $M_{\text {bol }}$ & $-7.0(0.2)$ & $-5.9(0.2)$ \\
\hline Abs. visual magnitude (mag) & $M_{\mathrm{v}}$ & $-3.9(0.2)$ & $-3.2(0.2)$ \\
\hline Bolometric correction (mag) & $B C$ & $-3.1(0.1)$ & $-2.7(0.1)$ \\
\hline Velocity amplitudes $\left(\mathrm{km} \mathrm{s}^{-1}\right)$ & $K_{1,2}$ & $211(3)$ & $292(5)$ \\
\hline Systemic velocity $\left(\mathrm{km} \mathrm{s}^{-1}\right)$ & $V_{\gamma}$ & \multicolumn{2}{|c|}{$-45.5(0.3)$} \\
\hline Observed rot. vel. $\left(\mathrm{km} \mathrm{s}^{-1}\right)$ & $\mathrm{V}_{\text {rot }}$ & $160(15)$ & $130(15)$ \\
\hline Computed sync. vel. $\left(\mathrm{km} \mathrm{s}^{-1}\right)$ & $\mathrm{V}_{\text {synch }}$ & $158(1)$ & $127(1)$ \\
\hline Distance (pc) & $d$ & \multicolumn{2}{|c|}{$1740(220)$} \\
\hline Proper motions ( $m a s \mathrm{yr}^{-1}$ ) & $\mu_{\alpha} \cos \delta, \mu_{\delta}$ & \multicolumn{2}{|c|}{$-2.57(1.60),-1.40(1.60)$} \\
\hline Space velocity components $\left(\mathrm{km} \mathrm{s}^{-1}\right)$ & $U, W, V$ & \multicolumn{2}{|c|}{$43.0(9.6),-21.8(9.2),-18.1(13.4)$} \\
\hline
\end{tabular}

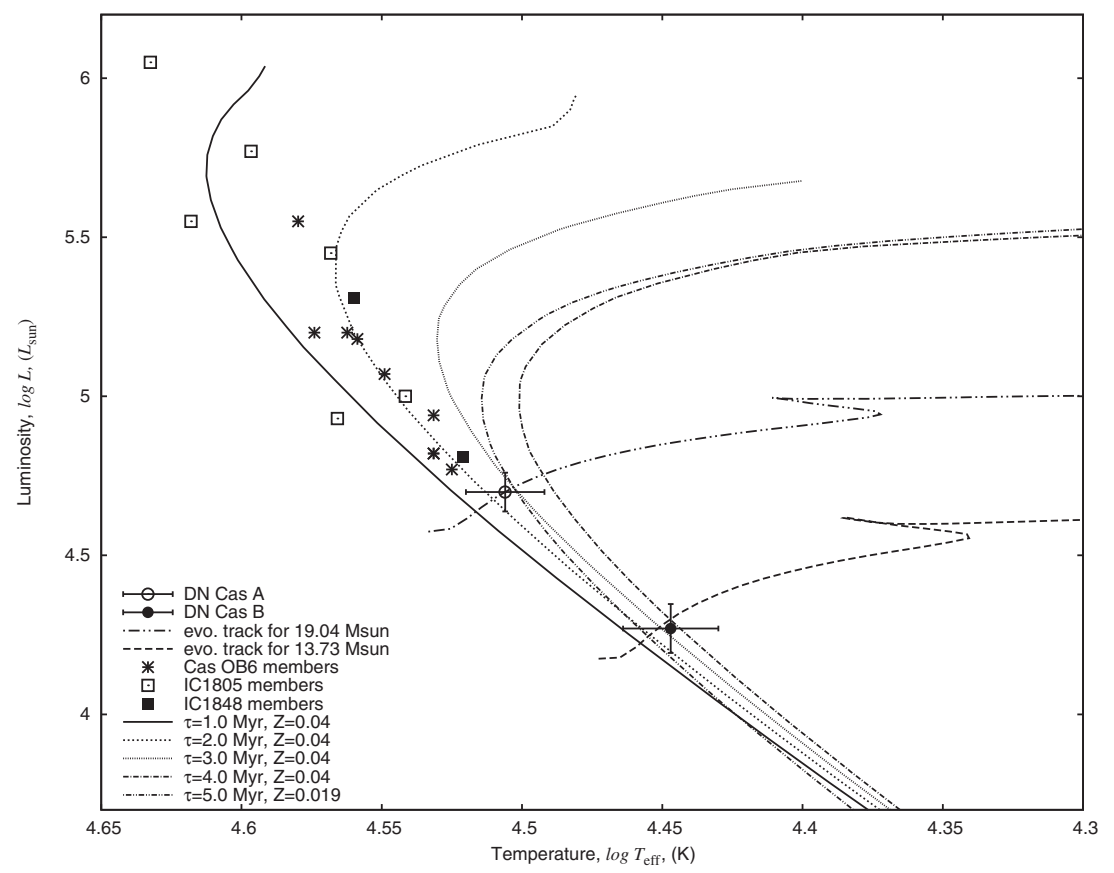

Figure 5. Location of DN Cas components in the $\log T_{\text {eff }}-\log L$ plane together with the members of two nearby open clusters (IC 1805 and IC 1848).

especially radial velocity measurements are needed to uncover the orbital eccentricity issue. One should note that a light curve alone does not provide a unique solution for $e$ and $\omega$ but $e \cos \omega$ for eclipsing binary systems (Kallrath \& Milone, 2009).

\subsection{Kinematical analysis and possible origin of DN Cas}

To analyse the kinematical and dynamical properties of DN Cas, we have used the system's proper motion, the distance, 
and the velocity of the centre-of-mass, which are given in Table 5. The proper motions were taken from the PPMXL Catalog of Positions and Proper Motions (Roeser, Demleitner, \& Schilbach 2010), whereas the centre-of-mass velocity and the distance are evaluated in this study. The system's space velocity with respect to Sun was calculated using Johnson \& Soderblom's (1987) algorithm where the space velocity components and their errors were found as $(U, V$, $W)=(43.0 \pm 9.6,-21.8 \pm 9.2,-18.1 \pm 13.4) \mathrm{km} \mathrm{s}^{-1}$ and given in Table 5. The first-order galactic differential rotation corrections were taken into account for precise space velocities, i.e. -34.1 and $-2.9 \mathrm{~km} \mathrm{~s}^{-1}$ for $U$ and $V$ space velocity components, respectively (Mihalas \& Binney 1981). The $W$ component is not affected from this first-order approximation. The velocity components are also corrected for the peculiar velocity of the Sun, which is $8.5 \pm 0.29,13.38 \pm 0.43,7 \pm 0.26$ $\mathrm{km} \mathrm{s}^{-1}$ given by Coşkunoğlu et al. (2011). Thus, the final LSR space velocity components $(U, V, W)_{\mathrm{LSR}}$ of DN Cas are $17.4 \pm 9.6,-5.5 \pm 9.2,-11.6 \pm 13.4 \mathrm{~km} \mathrm{~s}^{-1}$. So that the total space velocity of DN Cas is $S=21.6 \pm 18.9 \mathrm{~km} \mathrm{~s}^{-1}$. This value is in agreement with the space velocities of young thin-disc stars (Leggett 1992).

We also have performed test-particle integration in a Milky Way potentials of disc, halo, and bulge components. The galactic orbital parameters of the system were calculated within the integration time of 3 Gyr using the GALPY code of Bovy (2015). This integration time corresponds to about 15 revolutions around the Galactic centre, so that the orbital parameters could be determined reliably. The perigalactic $\left(R_{\min }\right)$ and apogalactic $\left(R_{\max }\right)$ distances of the system were found as 8.07 and $9.83 \mathrm{kpc}$, respectively. It turned out that the maximum distance of the system from the Galactic plane and the planer ellipticity of the system are $Z_{\max }=203 \mathrm{pc}$ and about $e_{p}=0.10$, respectively. These values show that DN Cas system is orbiting the Galaxy almost in a circular orbit and that the system belongs to the galactic thin-disc population.

We have also compared the kinematic data of $\mathrm{OB}$ associations and open clusters in the vicinity of the binary system in order to investigate the possible birthplace of DN Cas. There are two sources of kinematical data of associations, Mel'nik \& Dambis (2009) and Tetzlaff et al. (2010). The space velocity components given in these studies are almost the same. According to the galactic velocities, the DN Cas is in agreement with Cas OB1 and Cas OB6 within the uncertainty box. However, the distance of Cas OB6 is given as $1.8 \mathrm{kpc}$ by Meln'ik \& Dambis (2009) and as $2.4 \mathrm{kpc}$ by Tetzlaff et al. (2010), whilst the distance of Cas OB1 is given as $2 \mathrm{kpc}$ by Meln'ik \& Dambis (2010) and as $2 \mathrm{kpc}$ by Tetzlaff et al. (2010). Nevertheless, there are distance determinations of the embedded clusters (see Section 1) in Cas OB6 which is in between 1.9-2.0 kpc. Moreover, according to the partition of Meln'ik \& Dambis (2009) (see Figure 1), the direction of the binary goes through the Cas OB6 borders and $\sim 10^{\circ}$ far from the Cas OB1 association. Consequently, the possibility of Cas OB1 membership of DN Cas is weaker than its OB6 membership. Considering a similar radial extension of the Cas OB6 association with its tangential extension (234 pc) given by Tetzlaff et al. (2010), the photometric distance of DN Cas $(d=1.7 \pm 0.2 \mathrm{kpc})$ implies that DN Cas is within the borders of Cas OB6. Moreover, the age of DN Cas ( $\tau=3-5 \mathrm{Myr}$ ) which is 1-2 Myr older than the embedded open clusters in Cas OB6 is another supporting evidence for the Cas OB6 membership. Further evidence which would rely on accurately determined chemical composition is needed for a secure membership.

\section{CONCLUDING REMARKS}

The present study of DN Cas, which is one of the massive members of Cas OB6 association, could be concluded with the following remarks:

- The physical parameters of DN Cas are obtained with high precision. These parameters allowed us to derive reliable distance and age for the system.

- Higher accuracy radial velocity measurements are necessary to investigate further if the very small eccentricity found in this study for the close orbit is true or spurious.

- The radial velocities given by Hillwig et al. (2010) perfectly matches the spectroscopic orbit without nonKeplerian effects. However, the model constructed for the system in the present study requires departures from pure-Keplerian motion in the radial velocities during the eclipses. More reliable radial velocities are necessary to clarify this discrepancy.

- It has been shown that DN Cas has at least one physically bound distant companion with a minimum mass of $0.86 \mathrm{M}_{\odot}$ in a $P_{12}=42(9) \mathrm{yr}$ orbit.

- DN Cas is $\sim 1-2 \mathrm{Myr}$ older than some known clusters (IC 1805 and IC 1848) in Cas OB6 implying an age dispersion in stellar formation history of Cas OB6.

\section{ACKNOWLEDGMENTS}

The spectroscopic observations with TFOSC are granted by the TÜBITAK National Observatory with the project code 12ARTT150-255. This research has made use of 'Aladin sky atlas'developed at CDS, Strasbourg Observatory, France.

\section{REFERENCES}

Bakış, V., Hensberge, H., Demircan, O., Zejda, M., Bilir, S., \& Nitschelm, C. 2015, ACPS, 496, 189

Bertelli, G., Nasi, E., Girardi, L., \& Marigo, P. 2009, A\&A, 508, 355

Bik, A., et al. 2012, ApJ, 744, 13

Bonnarel, F., et al. 2000, A\&AS, 143, 33

Bovy, J. 2015, ApJS, 216, 29

Coşkunoğlu, B., et al. 2011, MNRAS, 412, 1237 
Davidge, T. J. 1980, IBVS, 1817, 1

Frazier, T. H., \& Hall, D. S. 1974, PASP, 86, 661

Hillwig, T. C., Gies, D. R., Bagnuolo, W. G. Jr., Huang, W., McSwain, M. V., \& Wingert, D. W. 2006, ApJ, 639, 1069

Hoffmeister, C. 1947, Veröff. Sternw. Sonneberg, 1, 81

Irwin, J. B. 1959, AJ, 64, 149

Johnson, D. R. H., \& Soderblom, D. R. 1987, AJ, 93, 864

Johnson, H. L., \& Morgan, W. W. 1953, ApJ, 117, 313

Kallrath, J., \& Milone, E. F. 2009, Eclipsing Binary Stars: Modeling and Analysis (New York: Springer-Verlag)

Kreiner, J. M., Kim, C.-H., \& Nha, Il-S. 2001, An Atlas of O-C Diagrams of Eclipsing Binary Stars (Cracow, Poland: Wydawnictwo Naukowe Akademii Pedagogicznej)

Leggett, S. K. 1992, ApJS, 82, 351

Lim, B., Sung, H., Kim, J. S., Bessell, M. S., \& Karimov, R. 2014, MNRAS, 438, 1451
Mason, B. D., Gies, D. R., Hartkopf, W. I., Bagnuolo, W. G. Jr., ten Brummelaar, T., \& McAlister, H. A. 1998, AJ, 115, 821

Meln'ik, A. M., \& Dambis, A. K. 2009, MNRAS, 400, 518

Mihalas, D., \& Binney, J. 1981, Galactic Astronomy: Structure and Kinematics (2nd edn.; San Francisco, CA: W. H. Freeman and Co.), 608

Özkardeş, B., Erdem, A., \& Bakış, V. 2009, NewA, 14, 461

Roeser, S., Demleitner, M., \& Schilbach, E. 2010, AJ, 139, 2440

Straižys, V., Boyle, R. P., Janusz, R., Laugalys, V., \& Kazlauskas, A. 2013, A\&A, 554, 3

Straižys, V., \& Kuriliene, G. 1981, Ap\&SS, 80, 353

Tetzlaff, N., Neuhäuser, R., Hohle, M. M., \& Maciejewski, G. 2010, MNRAS, 402, 2369

Wilson, R. E., \& Devinney, E. J. 1971, ApJ, 166, 605

Zahn, J. P. 1977, A\&A, 57, 383

Zakirov, M. M. 2001, KFTN, 17, 313 